\title{
Restoration of Forest Ecosystems on Disturbed Lands on the Northern Forest Distribution Border (North-East of European Russia)
}

\author{
Irina Likhanova and Inna Archegova \\ Institute of Biology Komi SC UrD RAS \\ Russia
}

\section{Introduction}

Since the second half of the $20^{\text {th }}$ century, due to active growth of minerals extraction in the European North of Russia, the area of disturbed forest ecosystems is steadily enlarges. It is known that disturbance of forest ecosystems leads to environmental unsteadiness and biodiversity decrease. It is vital to mention, that forest destruction negatively affects the traditional way of life of local people who depend on forest resources.

Natural forest destruction is a global problem. According to Losev K.S. et al. (2005) it is noticed that intensive growth of cultivated areas in the world is generally connected with forest destruction. About $63 \%$ of land on the planet is developed, what leads to infringement of balance stability if elements biogens (biological circulation of substances) and infraction of biosphere steadiness - habitat of human beings. It is noticed that in present time it is essential "to stop the destruction of this the most important ecological resource and then start natural restoration of forest ecosystems" (p. 78). It is vital for northern conditions, where forest ecosystems are particularly vulnerable to technogenic impact. Taiga forest ecosystems are easily disturbed and slowly self-recover after technogenic impact. This phenomenon was thoroughly surveyed by V.V. Ponomareva $(1970,1980)$. She wrote that forests are adjusted to strongly expressed eluvial conditions, “...forests minimize leaching of biophil elements from soil by accumulation them not in soil but in their huge always-living phytomass; (forests - ed.) exist from the autonomous above-soil circulation of elements between living organisms and their dying remnants which concentrate on soil surface" (Ponomareva, Plotnikova, 1980; P. 188). Thus, soils under taiga forests are characterized with high moisture content and so biological circulation in such ecosystems is nearer to an autonomous and close type. Huge perennial phytomass of tree plants holds organic elements it has assimilated from the upper earth's crust layer and partly gives them back with tree waste; then dead leaves and branches on soil surface are decomposed and provide for a new portion of nutrient for roots in soil litter. In this connection, forest soils have a very thin organic (productive) layer underlain by almost non-productive mineral layers with low absorbing capacity and containing practically none of plant nutrients. Technogenic interference easily destroys a thin organic soil layer and bares biologically inert mineral soil 
horizons which are not appreciated for plants. Restoration of disturbed forests on poor and strongly moist substratum is kind of difficult. In consequence, formerly forested areas undergo quick erosion and so their restoration further slows down.

The above-said information evidences a necessity for man-induced maintenance of ecosystem restoration on disturbed lands. In this article the results of investigation method are presented in comparative way. Method that accelerates the process of taiga forest ecosystem restoration is compared to traditional ways of forest cultivation.

\section{Objects and investigation methods}

We have studied different restoration modes of forest ecosystems in the Usinsk region of the Komi Republic (Russia). This particular region is characterized by severe climatic conditions. Annual air temperature is $-3.2{ }^{\circ} \mathrm{C}$. The coldest month is January with mean temperature $-18.4{ }^{\circ} \mathrm{C}$. Snow cover holds 200 days and is $48 \mathrm{~cm}$ high. The period with mean daily temperatures over $+5^{\circ} \mathrm{C}$ is 110 days. Mean air temperature in July, the warmest month, is $+13.8{ }^{\circ} \mathrm{C}$. Annual precipitation is $474 \mathrm{~mm}$, among them $159 \mathrm{~mm}$ precipitate during vegetation period (June-August) (Scientific-applied reference book..., 1989).

Principal vegetation of the study region, which is located in far north taiga subzone, is forests that intermixed with large marshes; $10 \%$ of the area is covered by tundra vegetation (Yudin, 1954). Dominant are forest stands sparse spruce and spruce-birch forests with crown density of $0.3-0.5$, height of trees 8-15 m, quality (bonitet) classes of tree stands V-Va. Forest composition mainly includes Pinus sylvestris and Larix sibirica. Most popular are long-moss forests, less represented are green-moss and sphagnum forest types.

The typical soil types of the region are boggy-podzolic, gley-podzolic, tundra-boggy, and boggy peaty soils (Podzolic soils..., 1981). Soil-forming rocks are moraine loams and sandy deposits formed in the glacier period. Sandy rocks are overlain by differently-moist soils as illuvial-humus-iron podzols, weakly peaty-podzolic-gley illuvial-humus soils; fine-textured loams are overlain by gley-podzolic soils and weakly peaty-podzolic-weakly gley soils.

The base of region's economy is oil-gas extracting and processing industries. Expansion of those industries leads to enlargement of the area of disturbed lands, including forest ecosystems. Sand pits are the most common technogenic objects within the Usinsk region. Sandy material excavated from pits is used for building roads, making bore sites and bore drills, etc. Restoration of vegetation cover in severe climatic conditions on sandy technogenic substrata, poor in nutrients, proceeds extremely slowly. Consequently, the restoration method of forest ecosystems on such lands is very much required for development.

Experimental plots were used in order to study the efficiency rate of forest ecosystems' restoration. Particularly, vegetation cover was studied using common geo-botanical methods (Field geo-botany, 1964), tree species that are planted on experimental plots were monitored by common study methods of forest cultures (Ogievskiy, Khirov, 1964). Soil type's description and soil samples' analysis (soil $\mathrm{pH}$, organic $\mathrm{C}$, exchangeable $\mathrm{Ca}, \mathrm{Mg}$, hydrolysable $\mathrm{N}, \mathrm{P}_{2} \mathrm{O}_{5}, \mathrm{~K}_{2} \mathrm{O}$ ) were done by the general methods (Agrochemical methods..., 1975; Theory and practice of soil chemical investigation, 2006). Humus composition was evaluated by the Tyurin method in Ponomareva-and-Plotnikova modification (1975). 


\section{Results and its discussion}

Investigations of new ways of forest ecosystem were done in a way of comparative analysis with traditional methods of forest recultivation.

Traditional restoration methods of forest ecosystems in the North The main methodological position of traditional restoration methods of disturbed forest ecosystems is the resource approach aiming at planting forest cultures of the principal forest-forming species as spruce and pine. In other words, the task of traditional technology is to create forest plantations and not to restore earlier destroyed forest ecosystem of previous quality (Losev et al., 2005). Coniferous cultures are planted at the age of 1-3 years and have open roots; no ground treatment is meant.

First forest cultures in the Usinsk region were planted in 1958 by the personnel of the Usinsk leskhoz. From 1991 to 2007 forest cultures were planted on area of 1020.8 ha. Among this figure, the share of Pinus sylvestris made 53.6\%, that of Picea obovata $27.6 \%$, and that of Pinus sibirica $1.5 \%$. Willow young trees were also planted for ground fixing with portion of $17.3 \%$. Most forest cultures (44.8\%) were planted on pits.

We have observed the sites being reforested by common restoration methods. The sites are located on the most usual sample of technogenic disturbance, in our case on $8 \mathrm{~b}$ technogenic pit (N 66 $\left.16^{\prime}, \mathrm{E} 57^{\circ} 16^{\prime}\right)$.

Sandy material on $8 \mathrm{~b}$ pit is characterized by low content of clay (sum of particles $<0.01$ is less than 6\%) (Table 1). This is responsible for low absorption and moisture content values; ground can be easily water- or wind-eroded.

\begin{tabular}{|c|c|c|c|c|c|c|c|c|c|c|}
\hline \multirow{2}{*}{$\begin{array}{c}\text { Sampling } \\
\text { depth } \\
(\mathrm{cm})\end{array}$} & \multirow{2}{*}{$\mid \begin{array}{c}\text { Hygroscopic } \\
\text { moisture, \% }\end{array}$} & \multirow{2}{*}{$\begin{array}{c}\mathrm{HCl} \\
\text { ignition } \\
\text { losses }\end{array}$} & \multicolumn{6}{|c|}{$\begin{array}{l}\text { Number of particles (\%) } \\
\text { with diameter of: }\end{array}$} & \multirow{2}{*}{$\begin{array}{c}\text { Sum of } \\
\text { particles } \\
>0.01\end{array}$} & \multirow{2}{*}{$\begin{array}{c}\text { Sum of } \\
\text { particles } \\
<0.01\end{array}$} \\
\hline & & & $\begin{array}{l}1.0- \\
0.25\end{array}$ & $\begin{array}{c}0.25- \\
0.05\end{array}$ & $\begin{array}{l}0.05- \\
0.01\end{array}$ & $\begin{array}{l}0.01- \\
0.005\end{array}$ & $\begin{array}{l}0.005- \\
0.001\end{array}$ & $<0.001$ & & \\
\hline $0-20$ & 0.39 & 0.00 & 11.35 & 81.88 & 0.66 & 1.47 & 0.30 & 4.34 & 93.89 & 6.11 \\
\hline $20-40$ & 0.43 & 0.37 & 11.94 & 80.59 & 2.11 & 0.04 & 0.64 & 4.67 & 94.64 & 5.36 \\
\hline
\end{tabular}

Table 1. Texture composition of technogenic material on $8 \mathrm{~b}$ pit.

The 8 " $\mathrm{b}$ " pit was partly planted with 3-year-old pine trees in 2001 without previous ground treatment.

On the second year after planting, the content of nitrogen, an important biogenic element, made $0.2 \mathrm{mg} / 100 \mathrm{~g}$ a.d.s. which corresponded with low organic carbon content (Table 2). On the eighth year, no significant quantitative changes in composition of nutrients and absorbed bases were observed; content of organic carbon resisted low.

Seven years after planting the survival rate of plantings made $50 \%$, tree height $53 \mathrm{~cm}$, and crown diameter $43 \mathrm{~cm}$. Above presented data is general. Pine plantings were underdeveloped because of poor concentration of nutrients in substratum and so were susceptible to the (snow) Schütte disease which stroke $60 \%$ of remaining pines. This disease additionally inhibits the growth of pines and often causes their depth. Soil cover was underdeveloped with total projection cover under $1 \% .7$ plant pioneers were identified (Festuca ovina, Chamaenerion angustifolium, Hieracium umbellatum, Equisetum arvense, Carex 
arctisibirica, Rumex acetosell), also in microdepressions mosses of the genus Polytrichum and Ceratodon purpureus, lichens of the Stereocaulon genus.

\begin{tabular}{|c|c|c|c|c|c|c|c|c|c|}
\hline \multirow{2}{*}{ Year } & \multirow{2}{*}{ Sampling depth, $\mathrm{cm}$} & \multirow{2}{*}{$\mathrm{pH}_{\text {water }}$} & \multirow{2}{*}{$\mathrm{C}_{\text {org., }} \%$} & \multicolumn{2}{|c|}{$\mathrm{N}_{\text {hygr. }}$} & $\mathrm{P}_{2} \mathrm{O}_{5}$ & $\mathrm{~K}_{2} \mathrm{O}$ & $\mathrm{Ca}^{2+}$ & $\mathrm{Mg}^{2+}$ \\
\cline { 5 - 9 } & & & \multicolumn{2}{|c|}{$\mathrm{mg} / 100 \mathrm{~g}$ a.d.s. } & \multicolumn{2}{l|}{$\mathrm{mM} / 100 \mathrm{~g}$ a.d.s. } \\
\hline \multirow{2}{*}{2002} & $0-10$ & 5.9 & 0.1 & 0.2 & 8.4 & 2.5 & 0.3 & 0.2 \\
\cline { 2 - 9 } & $10-20$ & 5.9 & 0.3 & 0.3 & 8.0 & 2.8 & 0.2 & 0.2 \\
\hline \multirow{2}{*}{2008} & $0-10$ & 6.0 & 0.3 & 0.1 & 9.4 & 2.3 & 0.3 & 0.2 \\
\cline { 2 - 9 } & $10-20$ & 5.9 & 0.4 & 0.4 & 7.9 & 3.1 & 0.2 & 0.2 \\
\hline
\end{tabular}

Table 2. Agrochemical indices of substratum planted with 3-year-old pine cultures.

Thus, unfavorable properties of ground material did not provide for the active self-restoring process.

The other common restoration method of disturbed area is planting willow. Willow cultures were planted on the above-mentioned 8 " $\mathrm{b}$ " pit under the leadership of the ecologist of the OSC "Northern Oil" V.I. Parfenyuk in 1991 without previous substratum treatment. The distance between plantings in a row was $25 \mathrm{~cm}$ and between rows $2 \mathrm{~m}$. On the twelfth planting year (2002) only $20 \%$ of planted trees remained alive and were about $1 \mathrm{~m}$ high. Single herbaceous plants Festuca ovina, Chamaenerion angustifolium, Hieracium umbellatum, Equisetum arvense were observed between rows. Those plant species are typical of the initial stage of selfrestoring succession (Table 3 ). Herbaceous layer projective cover made less than $1 \%$.

\begin{tabular}{|l|c|c|c|c|c|c|c|}
\hline Species & \multicolumn{3}{|c|}{ Availability, \% } & \multicolumn{3}{|c|}{ Projective cover, $\%$} & Height, cm \\
\hline Herbaceous plants: & 2002 & 2006 & 2011 & 2002 & 2006 & 2011 & \\
\hline Carex arctisibirica (Jurtz.) Czer. & - & 7 & 5 & - & $<1$ & $<1$ & 15 \\
\hline Chamaenerion angustifolium (L.) Scop & 16 & 29 & 20 & $<1$ & $<1$ & $<1$ & $20-30$ \\
\hline Equisetum arvense L. & 48 & 56 & 50 & 1 & $<1$ & $<1$ & 10 \\
\hline Festuca ovina L. & 25 & 53 & 60 & $<1$ & $<1$ & $<1$ & $10-25$ \\
\hline Hieracium umbellatum L. & 24 & 29 & 20 & $<1$ & $<1$ & $<1$ & $20-30$ \\
\hline Leucanthemum vulgare Lam. & 4 & - & - & $<1$ & - & - & 10 \\
\hline Rumex acetosella L. & 36 & 14 & 5 & $<1$ & $<1$ & $<1$ & $15-20$ \\
\hline Solidago virgaurea L. & - & 17 & 10 & - & $<1$ & $<1$ & $10-25$ \\
\hline $\begin{array}{l}\text { Tripleurospermum perforatum } \\
\text { (Merat.) M.Lainz }\end{array}$ & 8 & 7 & - & $<1$ & $<1$ & - & 10 \\
\hline Avenella flexuosa L. & - & - & 5 & - & - & $<1$ & 25 \\
\hline Mosses: & & & & & & & \\
\hline Bryum sp. & - & 7 & 5 & - & $<1$ & $<1$ & 1 \\
\hline Ceratodon purpureus (Hedw.) Brid. & - & 7 & 10 & - & $<1$ & 1 & 1 \\
\hline Polytrichum piliferum Hedw. & - & 7 & 60 & - & $<1$ & 5 & 1 \\
\hline Lichens: & & & & & & & \\
\hline Stereocaulon paschale (L.) Hoffm. & - & 7 & 15 & - & $<1$ & $<1$ & $1-2$ \\
\hline Cladonia sp. & - & - & 7 & - & - & $<1$ & $1-2$ \\
\hline
\end{tabular}

Note: «-» - not found.

Table 3. Species composition of soil cover at site planted with willow trees. 
20 years after planting (2011), the health status of shrubby layer did not practically change; soil cover remained thin (Table 3 ). The surface of ground material was partly covered with algae film and protonema of mosses. Microdepressions hosted mosses of the Polytrichum genus and Ceratodon purpureus. Totally, 8 species of herbaceous plants and 3 mosses were identified at the area.

Chemical analysis of ground material samples on the twelfth willow planting year revealed a low content of biogenic elements and organic matter. On the sixteenth planting year the agrochemical parameters did not practically change (Table 4). As the herbaceous layer was very thin, sandy ground was susceptible to erosion. On the twelfth planting year we observed washed-out erosion hollow $2.5 \mathrm{~m}$ wide, $1 \mathrm{~m}$ deep, $5 \mathrm{~m}$ long. On the sixteenth planting year it increased in size with a depth of $1.5 \mathrm{~m}$ and a length over $10 \mathrm{~m}$.

\begin{tabular}{|c|c|c|c|c|c|c|c|c|c|}
\hline \multirow{2}{*}{ Year } & \multirow{2}{*}{$\begin{array}{c}\text { Sampling } \\
\text { depth, cm }\end{array}$} & \multirow{2}{*}{$\mathrm{pH}_{\text {water }}$} & \multirow{2}{*}{$\mathrm{C}_{\text {org., }} \%$} & \multicolumn{2}{|c|}{$\mathrm{N}_{\text {hygr. }}$} & $\mathrm{P}_{2} \mathrm{O}_{5}$ & $\mathrm{~K}_{2} \mathrm{O}$ & $\mathrm{Ca}^{2+}$ & $\mathrm{Mg}^{2+}$ \\
\cline { 5 - 10 } & & \multicolumn{2}{|c|}{$\mathrm{mg} / 100 \mathrm{~g}$ a.d.s. } & \multicolumn{2}{c|}{$\mathrm{mM} / 100$ g a.d.s. } \\
\hline \multirow{2}{*}{2002} & $0-10$ & 5.9 & 0.2 & 0.1 & 9.4 & 2.3 & 0.3 & 0.2 \\
\cline { 2 - 9 } & $10-20$ & 5.9 & 0.2 & 0.4 & 7.8 & 3.1 & 0.2 & 0.2 \\
\hline 2005 & $0-2^{*}$ & 5.9 & 1.2 & 1.7 & 9.7 & 0.7 & 1.4 & 0.2 \\
\hline 2006 & $0-5$ & 5.8 & 0.2 & 0.1 & 7.9 & 6.5 & 0.8 & 0.6 \\
\hline
\end{tabular}

Note: * - crust of algae and protonema in a small depression.

Table 4. Agrochemical parameters of ground material planted with willow trees.

Consequently, there was almost no positive effect from willow planting for posttechnogenic substratum restoration.

The above-sited data allow for the following conclusion. While using the traditional restoration methods of disturbed forested areas, soil and vegetation cover formation is slow, what hampers restoration of the forest ecosystem as whole. To speed up restoration there is a need to apply complex methods aiming at development plant biogeocenosis, i.e. maintenance plant matter biological cycle, ensuring conditions for intensive plant cover formation on ground surface and organic matter accumulation in substratum. So, restoration the technogenically disturbed forest ecosystem in the North cannot be efficient without development the basic system components, first of all plant community including not only tree layer but also ground plant cover.

\section{Main principles of the "nature restoration" conception and the complex of methods aimed at accelerated restoration of forest ecosystems on technogenically disturbed lands}

The "nature restoration" conception was developed at the Institute of Biology Komi SC UrD RAS under the leadership of Dr. I.B. Archegova. This conception means restoration of forest ecosystems including their initial structure and "functions" which, finally, ensures the integrity of the biosphere (Archegova, 1998). The "nature restoration" methodological conception operates forest as a system and ecosystem self-restoration as a succession process. From this point of view, ecosystem presents a system of the three main components as plant community, fauna-microbe complex that processes plant remnants and soil that is a productive (biogenic-accumulative) layer. These three components are integrated into ecosystem by means of organic (plant) matter biological cycle. In practice, the "nature restoration" system aims at restoration the ecosystem as a whole, not its single components, 
tree layer in particular. The "nature restoration" methods should correspond with the regional climatic conditions, also taking into consideration regional economy, traditional regional nature management.

Northern ecosystems poorly resist technogenic impacts and slowly self-restore because of not only severe climatic conditions together with the presence of permafrost rocks but also because of a thin productive organic-accumulative layer (soil) which hosts the majority of nutrition elements, plant roots, and active microbiota. Mineral layer becomes visual after organic-accumulative layer disturbance. Mineral layer is unfavorable for biota selfrestoration and so hampers the process of nature self-restoration, first of all plant community restoration. Consequently, any organic layer technogenic disturbance always has total nature ecosystem destruction as an aftereffect. The absence of vegetation cover for a long period of time speeds up erosion processes that only aggravate self-restoration of plant-soil cover. This situation requires development an active and up-to-date approach to restoration of disturbed forest ecosystems.

Based on the "nature restoration" conception we have developed the two-stage system of rapid (managed) "nature restoration" practical methods (Fig. 1). At the first "intensive" stage, we form herbaceous ecosystem and corresponding biogenic-accumulative layer in a short period of time, namely in 3-5 years, using complex agrotechnical treatments as applying organic and mineral fertilizers and sowing local perennial herbs. In the other words, this way reduces the usually long (up to 30-40 years) initial self-restoration succession stage. At the second "assimilation" stage, no agrotechnical treatments are used. The previously formed herbaceous community is gradually self-replaced by a zonal type of plant community or generally by forest ecosystem.

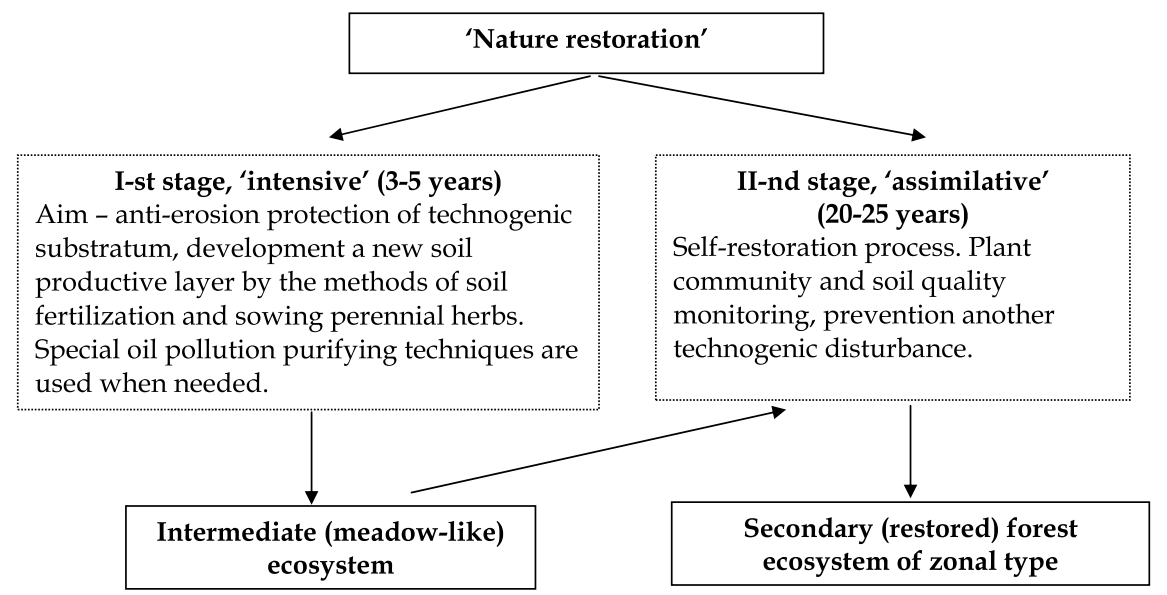

Fig. 1. The scheme of complex 'nature restoration' methods.

According to this sketch in 1991 we initiated the experiment on the 8 " $\mathrm{b}$ " pit located near the above discussed plots being restored by traditional methods. Intensive "nature restoration" methods included soil surface fertilization with peat in a $15-\mathrm{cm}$ layer and with mineral fertilizers $\left(\mathrm{N}_{60} \mathrm{P}_{60} \mathrm{~K}_{60}\right)$, sowing the herbaceous mixture of Alopecurus pratensis and Poa pratensis in a dose of $20 \mathrm{~kg}$ seeds/ha with proportion of seeds 1:1. Initial sandy substratum 
contained $0.2 \%$ organic carbon, 0.1 hydrolysable nitrogen, 6.7 phosphorus and $2.0 \mathrm{mg} / 100 \mathrm{~g}$ a.d.s. potassium oxides. Sawn grasses were annually given a complex mineral fertilizer in a rate of $30 \mathrm{~kg}$ each mineral element/ha.

Three years later the plot grew into an intermediate herbaceous community (TPC $100 \%$ ) of meadow type with corresponding meadow-like soil constituted of typical soddy horizon and humus horizon. Later there was no any kind of treatment. It was the beginning of the second restoration stage thereby tree plants inhabited the plot with gradual replacement of herbaceous ecosystem to forest ecosystem.

After intensive agrotechnical treatments, already the first ten years saw formation of woodyshrubby layer with a crown density of 0.1 of Betula pubescens, Larix sibirica, Salix species 1.5$2.5 \mathrm{~m}$ high (Table 5). Ground cover (TPC 100\%) 10 years after restoration start was considerably composed of sawn Alopecurus pratensis (PC 44\%) and less by Poa pratensis. At the same time, 19 new non-sown herbaceous species were observed. Among them, Festuca ovina, Chamaenerion angustifolium, Erigeron acris, and Solidago virgaurea had highest projective cover figures (Table 8). Ground cover was largely composed of synanthropic species (Rumex acetosella, Chamaenerion angustifolium, Crepis tectorum, Tripleurospermum perforatum, Tussilago farfara, Equisetum arvense), characteristic of initial restoration stages of disturbed lands. The plot was inhabited by mosses and single Cladonia and Peltigera lichens; 5 mosses were totally identified dominated by polytrichum mosses (16\%) (Table 8). Consequently, the first ten years after the experiment start there is an active replacement of intermediate herbaceous ecosystem by forest ecosystem with formation of woody layer of quickly-growing tree species. Ground cover was identified for numerous non-sown vascular plant species and still numerous sawn grasses and mosses.

\begin{tabular}{|c|c|c|c|c|}
\hline Species & \multicolumn{2}{|c|}{ Quantity, inds./100 $\mathrm{m}^{2}$} & \multicolumn{2}{|c|}{ Height, $\mathrm{m}$} \\
\hline & 2002 & 2011 & 2002 & 2011 \\
\hline \multicolumn{5}{|c|}{ Developing tree story: } \\
\hline \multicolumn{5}{|c|}{ 1st layer: } \\
\hline Betula pubescens Ehrh. & 2 & 13 & $2.5-3$ & $4-6$ \\
\hline Larix sibirica Ledeb. & 9 & 15 & $1.3-1.5$ & $4-5,5$ \\
\hline Salix caprea L. & 2 & 2 & 2.5 & 6 \\
\hline Salix dasyclados Wimm. & 2 & 1 & 3 & 4 \\
\hline \multicolumn{5}{|c|}{ 2nd layer: } \\
\hline Betula pubescens Ehrh. & - & 16 & - & $2-3$ \\
\hline Larix sibirica Ledeb. & - & 6 & - & $2-3$ \\
\hline \multicolumn{5}{|c|}{ 3rd layer: } \\
\hline Betula pubescens Ehrh. & - & 13 & - & $0.5-1.5$ \\
\hline Larix sibirica Ledeb. & - & 3 & - & $0.5-1.5$ \\
\hline Picea obovata Ledeb. & - & 1 & - & 0,5 \\
\hline \multicolumn{5}{|c|}{ Developing tree understory: } \\
\hline Betula nana L. & 1 & 1 & 0.7 & $1-1.5$ \\
\hline Ribes rubrum L. & 1 & 1 & 0.7 & 1 \\
\hline Salix phylicifolia L. & 11 & 6 & 1.5 & $1-3$ \\
\hline Salix hastata L. & 1 & - & 0.9 & - \\
\hline Salix lapponum L. & 1 & - & 1.3 & - \\
\hline
\end{tabular}

Table 5. Species composition and structure of tree story and tree understory on the experimental plot. 


\begin{tabular}{|c|c|c|c|c|c|c|c|}
\hline \multirow{2}{*}{ Species } & \multicolumn{3}{|c|}{ Abundance, $\%$} & \multicolumn{3}{|c|}{ Projective cover, $\%$} & \multirow{2}{*}{ Height, $\mathrm{cm}$} \\
\hline & 2002 & 2006 & 2011 & 2002 & 2006 & 2011 & \\
\hline \multicolumn{8}{|l|}{ Shrubs: } \\
\hline Arctostaphylos uva-ursi (L.) Spreng. & 5 & 7 & 5 & $<1$ & $<1$ & $<1$ & 10 \\
\hline $\begin{array}{l}\text { Empetrum hermaphroditum (Lange) } \\
\text { Hagerup }\end{array}$ & 5 & 7 & 10 & $<1$ & $<1$ & $<1$ & 10 \\
\hline Vaccinium uliginosum L. & - & - & 5 & - & - & $<1$ & 10 \\
\hline Vaccinium myrtillus L. & - & - & 5 & - & - & $<1$ & 10 \\
\hline \multicolumn{8}{|l|}{ Herbaceous plants: } \\
\hline Agrostis tenuis Sibth. & - & 7 & 35 & - & $<1$ & 5 & $20-50$ \\
\hline Avenella flexuosa (L.) Drey. & - & - & 70 & - & - & 25 & $20-50$ \\
\hline Alopecurus pratensis L. & 100 & 80 & 75 & 44 & 46 & 25 & 80 \\
\hline Antennaria dioica (L.) Gaertn. & - & - & 5 & - & - & $<1$ & 15 \\
\hline Calamagrostis epigeios (L.) Roth & 10 & 7 & - & $<1$ & $<1$ & - & $60-80$ \\
\hline Calamagrostis lapponica (Wahl.) Hartm. & - & - & 15 & - & - & 1 & $50-60$ \\
\hline Carex brunnescens (Pers.) Poir & 5 & - & 5 & $<1$ & - & $<1$ & 25 \\
\hline Chamaenerion angustifolium (L.) Scop. & 100 & 67 & 65 & 9 & 5 & 7 & $40-80$ \\
\hline Crepis tectorum L. & 5 & 7 & & $<1$ & $<1$ & & $20-25$ \\
\hline Deschampsia cespitosa (L.) Beauv. & 30 & 7 & 25 & 2 & 1 & 7 & $40-80$ \\
\hline Epilobium palustre L. & 10 & 7 & - & $<1$ & $<1$ & - & 20 \\
\hline Equisetum arvense L. & 10 & 7 & 15 & $<1$ & $<1$ & $<1$ & 20 \\
\hline Equisetum sylvaticum L. & 5 & - & & $<1$ & - & - & 25 \\
\hline Euphrasia frigida Pugsl. & - & - & 5 & - & - & $<1$ & 15 \\
\hline Erigeron acris L. & 70 & 40 & 40 & 4 & $<1$ & 1 & $35-45$ \\
\hline Festuca ovina L. & 100 & 67 & 40 & 23 & 18 & 16 & $15-30$ \\
\hline Festuca rubra L. & - & 7 & 15 & - & $<1$ & 1 & $40-45$ \\
\hline Hieracium umbellatum L. & 40 & 33 & 25 & 1 & 1 & 1 & 40 \\
\hline Hieracium vulgatum L. & 10 & 20 & 10 & $<1$ & 1 & $<1$ & 40 \\
\hline Omalotheca sylvatica (L.) Sch.Bip. & 60 & 33 & 70 & 2 & 1 & 2 & $10-30$ \\
\hline Phalaroides arundinacea (L.) Rausch. & - & - & 5 & - & - & $<1$ & 50 \\
\hline Poa pratensis L. & 30 & 20 & 30 & 1 & 4 & 5 & 60 \\
\hline Rumex acetosella L. & 10 & 7 & 25 & $<1$ & $<1$ & $<1$ & $20-25$ \\
\hline Solidago virgaurea L. & 80 & 60 & 80 & 3 & 3 & 10 & $10-45$ \\
\hline Taraxacum officinale Wigg. & 40 & 20 & 5 & 1 & $<1$ & $<1$ & $15-20$ \\
\hline $\begin{array}{l}\text { Tripleurospermum perforatum (Merat.) } \\
\text { M.Lainz }\end{array}$ & 5 & 7 & - & $<1$ & $<1$ & - & 15 \\
\hline Tussilago farfara $\mathrm{L}$. & 20 & 7 & 5 & $<1$ & $<1$ & $<1$ & $10-15$ \\
\hline Trientalis europaea L. & - & - & 5 & - & - & $<1$ & $5-10$ \\
\hline Lycopodium annotinum L. & - & - & 5 & - & - & $<1$ & 10 \\
\hline Lathyrus pratensis L. & - & - & 5 & - & - & $<1$ & $30-40$ \\
\hline Orthilia secunda (L.) House & - & - & 5 & - & - & $<1$ & 15 \\
\hline \multicolumn{8}{|l|}{ Mosses: } \\
\hline Brachythecium campestre (Bruch) B. S. G. & - & - & 20 & - & - & 5 & $3-5$ \\
\hline Brachythecium reflexum (Starke) Schimp. & - & - & 10 & - & - & 5 & $3-5$ \\
\hline $\begin{array}{l}\text { Brachythecium salebrasum (Wed et Mohr) } \\
\text { Bryol. }\end{array}$ & - & - & 20 & - & - & 5 & $3-5$ \\
\hline
\end{tabular}




\begin{tabular}{|c|c|c|c|c|c|c|c|}
\hline \multirow{2}{*}{ Species } & \multicolumn{3}{|c|}{ Abundance, $\%$} & \multicolumn{3}{|c|}{ Projective cover, $\%$} & \multirow{2}{*}{ Height, $\mathrm{cm}$} \\
\hline & 2002 & 2006 & 2011 & 2002 & 2006 & 2011 & \\
\hline Brachythecium sp. & 20 & 20 & - & 1 & 15 & - & $3-5$ \\
\hline Ceratodon purpureus (Hedw.) Brid. & 20 & 20 & 5 & $<1$ & $<1$ & $<1$ & $2-3$ \\
\hline Dicranum polysetum Sw. & - & - & 10 & - & - & 1 & $3-4$ \\
\hline Plagiothecium denticulatum (Hedw.) B. S. G. & - & - & 10 & - & - & 1 & $1-2$ \\
\hline Pleurozium schreberi (Brid.) Mitt. & - & 20 & 80 & - & 1 & 10 & $3-5$ \\
\hline Polytrichum commune Hedw. & 20 & 20 & 20 & 2 & 1 & 2 & $5-7$ \\
\hline Polytrichum juniperinum Hedw. & 60 & 40 & 90 & 11 & 11 & 30 & $5-7$ \\
\hline Polytrichum piliferum Hedw. & 30 & 33 & 15 & 5 & 5 & 3 & $3-5$ \\
\hline $\begin{array}{l}\text { Sciurohypnum oedipodium (Mitt.) Ignatov } \\
\text { et Huttunen. }\end{array}$ & - & - & 10 & - & - & 1 & 3 \\
\hline $\begin{array}{l}\text { Sciuro-hypnum starkei (Brid.) Ignatov et } \\
\text { Huttunen (Brachythecium starkei(Brid.) } \\
\text { B.S.G.) }\end{array}$ & - & - & 65 & - & - & 30 & $3-5$ \\
\hline \multicolumn{8}{|l|}{ Lichens: } \\
\hline Cladonia anomaea (Ach.) Ahti \& P.James & - & - & 4 & - & - & $<1$ & $1-3$ \\
\hline Cladonia arbuscula (Wallr.) Flot. & - & 7 & 10 & - & $<1$ & $<1$ & $1-3$ \\
\hline Cladonia borealis Stenroos & - & - & 4 & - & - & $<1$ & $1-2$ \\
\hline Cladonia botrytes (Hag.) Willd. & - & - & 4 & - & - & $<1$ & 1 \\
\hline Cladonia carneola (Fr.) Fr. & - & 7 & - & - & $<1$ & - & $1-2$ \\
\hline $\begin{array}{l}\text { Cladonia cervicornis (Ach.) Flot. ssp. } \\
\text { verticillata (Hoffm.) }\end{array}$ & - & - & 4 & - & - & $<1$ & $1-2$ \\
\hline $\begin{array}{l}\text { Cladonia chlorophaea (Florke ex Sommerf.) } \\
\text { Spreng. }\end{array}$ & - & - & 4 & - & - & $<1$ & $1-2$ \\
\hline Cladonia cornuta (L.) Hoffm. & - & 7 & 20 & - & $<1$ & $<1$ & $1-4$ \\
\hline Cladonia crispata (Ach.) Flot. & - & - & 4 & - & - & $<1$ & $1-2$ \\
\hline Cladonia deformis (L.) Hoffm. & - & 7 & - & - & $<1$ & $<1$ & $1-3$ \\
\hline Cladonia fimbriata (L.) Fr. & - & 7 & 10 & - & $<1$ & $<1$ & $1-3$ \\
\hline Cladonia gracilis (L.) Willd. & - & 7 & 20 & - & $<1$ & $<1$ & $1-3$ \\
\hline Cladonia phylophora Hoffm. & - & - & 20 & - & - & $<1$ & $1-2$ \\
\hline Cladonia pleurota (Floerke) Schaer. & - & - & 4 & - & - & $<1$ & $1-2$ \\
\hline Cladonia rangiferina (L.) Web. & - & 7 & 20 & - & $<1$ & $<1$ & $1-3$ \\
\hline Cladonia subulata Weber. & - & - & 4 & - & - & $<1$ & $1-2$ \\
\hline Cladonia sp. & 10 & 7 & - & $<1$ & $<1$ & - & 1 \\
\hline Peltigera didactyla (With.) Laundon & - & - & 10 & - & - & $<1$ & $1-2$ \\
\hline Peltigera leucophlebia (Nyl.) Gyeln. & - & - & 4 & - & - & $<1$ & $1-2$ \\
\hline Peltigera rufescens (Weis.) Humb. & - & - & 4 & - & - & $<1$ & $1-2$ \\
\hline Peltigera sp. & 10 & 7 & - & $<1$ & - & $<1$ & $1-2$ \\
\hline
\end{tabular}

Table 6. Composition and structure of ground plant cover on the experimental plot.

The newly-formed 10-year-old soil had the following morphological structure. Loose layer of weakly-decomposed plant waste (dead grass) was penetrated with rare moss stems. 
AOA1 0-8(12) cm

A1 layer 8(12)-21 cm

III-rd layer $21-29 \mathrm{~cm}$

IV-th layer $29-45 \mathrm{~cm}$
Well-decomposed plant waste with inclusions of mineral particles, moist, abundant roots.

Sandy, dark-grey (humus color), structureless, loose, moist, many roots. Transition to the next horizon is abrupt by color.

Sandy, light-yellowish with whitish and dark-ochre spots, loose, moist, rare roots.

Sandy, grey-yellowish, lighter than the previous horizon, loose, moist, without roots.

In the first ten years there is a soil profile formation with organic horizons with features of soddy layer, typical for meadow ecosystems. The upper biogenic-accumulative layers (A0A1, A1) had weakly-acid medium reaction and accumulated the maximum of nitrogen, humus, and exchangeable bases (Table 7).

\begin{tabular}{|c|c|c|c|c|c|c|c|c|c|}
\hline \multirow[t]{2}{*}{ Year } & \multirow{2}{*}{\multicolumn{2}{|c|}{$\begin{array}{c}\text { Horizon, } \\
\text { sampling depth, } \\
\mathrm{cm}\end{array}$}} & \multirow[t]{2}{*}{$\mathrm{pH}_{\text {water }}$} & \multirow[t]{2}{*}{ C, \% } & $\begin{array}{c}\mathrm{N}_{\text {hydr }} \\
\text {. }\end{array}$ & $\mathrm{P}_{2} \mathrm{O}_{5}$ & $\mathrm{~K}_{2} \mathrm{O}$ & $\mathrm{Ca}^{2+}$ & $\mathrm{Mg}^{2+}$ \\
\hline & & & & & \multicolumn{3}{|c|}{ mg/100 g a.d.s. } & \multicolumn{2}{|c|}{ mM/100 g a.d.s. } \\
\hline \multirow{4}{*}{$\begin{array}{l}11 \text { th } \\
\text { year } \\
(2002)\end{array}$} & A0A1 & $0-8(12)$ & 5.4 & 5.0 & 2.9 & 8.5 & 7.9 & 7.1 & 1.1 \\
\hline & A1 & $8(12)-21$ & 5.7 & 3.3 & 1.6 & 10.1 & 4.3 & 5.9 & 0.8 \\
\hline & III & $21-29$ & 5.7 & 1.6 & 0.8 & 9.9 & 3.8 & 1.7 & 0.5 \\
\hline & IV & $29-45$ & 5.6 & 0.2 & 0.8 & 10.0 & 2.5 & 1.2 & 0.4 \\
\hline \multirow{11}{*}{$\begin{array}{l}20 \text { th } \\
\text { year } \\
(2011)\end{array}$} & \multicolumn{9}{|c|}{ In group of trees } \\
\hline & A0 & $0-5$ & 5.4 & 6.7 & 11.5 & 10.0 & 29.0 & 17.7 & 3.6 \\
\hline & A1 & $5-15$ & 5.2 & 4.9 & 9.0 & 9.9 & 9.0 & 10.0 & 1.6 \\
\hline & $\mathrm{A} 0_{\text {buried }}{ }^{*}$ & $15-21$ & 5.0 & $7.2^{*}$ & 5.9 & 8.3 & 8.9 & 14.8 & 2.1 \\
\hline & $\mathrm{BC}$ & $21-35$ & 5.3 & 0.2 & 7.8 & 14.0 & 8.6 & 2.3 & 0.6 \\
\hline & \multicolumn{9}{|c|}{ Open area } \\
\hline & A0 & $0-2(4)$ & 5.3 & 5.7 & 9.5 & 13.3 & 36.0 & 10.6 & 2.2 \\
\hline & $\mathrm{A}_{1} \quad 2(4)$ & )$-13,5(14)$ & 4.9 & 5.4 & 5.9 & 11.1 & 11.4 & 10.1 & 1.6 \\
\hline & $\begin{array}{r}\mathrm{A} 0 \text { buried } \\
2\end{array}$ & $\begin{array}{l}13,5(14)- \\
11\end{array}$ & 4.9 & $14.8^{*}$ & 5.5 & 5.3 & 14.9 & 17.5 & 2.2 \\
\hline & $\overline{B C 2}$ & $21-24$ & 4.8 & 0.3 & 2.4 & 12.8 & 5.5 & 2.5 & 0.6 \\
\hline & $\mathrm{BC} / 2$ & $24-35$ & 5.1 & 0.2 & 1.1 & 13.2 & 3.8 & 2.4 & 0.6 \\
\hline
\end{tabular}

Note: * - buried organic residues (peat).

Table 7. Agrochemical indices of newly-formed soil on the experimental plot.

The forest community was structurally formed on the 20th year after restoration start. The first tree story consisted of Betula pubescens and Larix sibirica with few tree-like willow species (Salix dasyclados, Salix caprea); tree height was 4-6 m, stem diameter 4-6(9) cm (Table 5). Crown density increased to 0.4. Since 2002, tree re-growth (with a height of less than 50 $\mathrm{cm}$ ) was clearly dominated by Betula pubescens, 40 individuals per $100 \mathrm{~m}^{2}$, for contrast only 5 individuals of Larix sibirica. Betula pubescens was also a dominant species in the second and third tree stories which were formed on the 20th restoration year. The plant waste accumulation dynamics of woody plants evidenced the active development of tree layer (Tables 8,9 ). Thus, the community on its 20th restoration year was at the stage of quicklygrowing woody plants, typical of self-restoration succession in the taiga zone (Shennikov, 
1964). Low-height young growth of Picea obovata and Pinus sylvestris appeared in amount of 1-3 individuals / $100 \mathrm{~m}^{2}$.

\begin{tabular}{|l|c|}
\hline \multicolumn{1}{|c|}{ Plant waste sampling period } & Plant waste weight \\
\hline June 2002 - September 2002 & $8.5 \pm 1.7$ \\
\hline October 2002 - May 2003 & $27.5 \pm 2.0$ \\
\hline Year total: & 36.1 \\
\hline June 2007 - September 2007 & $15.0 \pm 6.5$ \\
\hline October 2007 - May 2008 & $52.2 \pm 8.6$ \\
\hline Year total: & 67.2 \\
\hline June 2008 - September 2008 & $13.6 \pm 5.4$ \\
\hline October 2008 - May 2009 & $53.05 \pm 10.2$ \\
\hline Year total: & 66.65 \\
\hline June 2009 - September 2009 & $11.9 \pm 2.8$ \\
\hline October 2009 - May 2010 & $73.3 \pm 15.3$ \\
\hline Year total: & 85.2 \\
\hline June 2010 - September 2010 & $14.7 \pm 3.2$ \\
\hline October 2010 - May 2011 & $106.01 \pm 31.2$ \\
\hline Year total: & 110.71 \\
\hline
\end{tabular}

Table 8. Plant waste weight by years on the experiment plot (air-dried weight, $\mathrm{g} / \mathrm{m}^{2}$ ).

\begin{tabular}{|l|c|c|c|c|c|c|c|c|c|c|}
\hline & \multicolumn{2}{|c|}{$\begin{array}{c}\text { October } 2008 \\
- \text { May 2009 }\end{array}$} & $\begin{array}{c}\text { June 2009 - } \\
\text { September } \\
2009\end{array}$ & \multicolumn{2}{|c|}{$\begin{array}{c}\text { October 2009 } \\
- \text { May 2010 }\end{array}$} & \multicolumn{2}{|c|}{$\begin{array}{c}\text { June 2010 - } \\
\text { September } \\
2010\end{array}$} & \multicolumn{2}{|c|}{$\begin{array}{c}\text { October } 2010 \\
- \text { May } 2011\end{array}$} \\
\hline Fraction & $\begin{array}{c}\text { Weight } \\
\mathrm{g} / \mathrm{m}^{2}\end{array}$ & $\begin{array}{c}\text { Share } \\
\%\end{array}$ & $\begin{array}{c}\text { Weight } \\
\mathrm{g} / \mathrm{m}^{2}\end{array}$ & $\begin{array}{c}\text { Share } \\
\%\end{array}$ & $\begin{array}{c}\text { Weight } \\
\mathrm{g} / \mathrm{m}^{2}\end{array}$ & $\begin{array}{c}\text { Share } \\
\%\end{array}$ & $\begin{array}{c}\text { Weight } \\
\mathrm{g} / \mathrm{m}^{2}\end{array}$ & $\begin{array}{c}\text { Share, } \\
\%\end{array}$ & $\begin{array}{c}\text { Weight } \\
\mathrm{g} / \mathrm{m}^{2}\end{array}$ & $\begin{array}{c}\text { Share, } \\
\%\end{array}$ \\
\hline Branches & 0.45 & 1 & 0.498 & 4 & 0.604 & 1 & 0.756 & 5 & 1.96 & 2 \\
\hline $\begin{array}{l}\text { Betula } \\
\text { pubescens } \\
\text { leaves }\end{array}$ & 30.68 & 58 & 2.263 & 19 & 35.88 & 49 & 1.028 & 7 & 68.59 & 65 \\
\hline Herbs & 2.17 & 4 & 0.169 & 1 & 3.664 & 5 & 0.412 & 3 & 2.80 & 3 \\
\hline Salix leaves & 2.48 & 5 & 4.421 & 37 & 1.972 & 3 & 0.780 & 5 & 2.95 & 3 \\
\hline Bark & 0.43 & 1 & & & 0.14 & 0 & 0.132 & 1 & 0.37 & 0 \\
\hline $\begin{array}{l}\text { Larix sibirica } \\
\text { needles }\end{array}$ & 8.46 & 16 & 1.046 & 9 & 21.352 & 29 & 9.792 & 67 & 20.50 & 19 \\
\hline $\begin{array}{l}\text { Dust of } \\
\text { rotten wood }\end{array}$ & 8.38 & 16 & 3.54 & 30 & 9.736 & 13 & 1.848 & 13 & 8.72 & 8 \\
\hline Inflorescences & - & - & - & - & - & - & - & - & 0.11 & 0 \\
\hline
\end{tabular}

Note: «-» - not found.

Table 9. Plant waste fraction composition on the experiment plot (air-dried weight, $\mathrm{g} / \mathrm{m}^{2}$ ).

At the end of the second decade of experimental years the TPC of herbaceous-dwarfshrub layer comprised $85 \%$. This retreat in TPC was related to woody plants' shadowing. Forest 
dwarfshrubs increased in species number, among them Arctostaphylos uva-ursi, Empetrum hermaphroditum, Vaccinium uliginosum, Vaccinium myrtillus (Table 6). Among 24 herbaceous species found on the experimental plot, the forest species Avenella flexuosa and Solidago virgaurea had essential PC, 25 and 10\%, correspondingly. The sawn meadow grass Alopecurus pratensis significantly reduced its PC (25\%). Mosses counted 11 species on the 19th experimental year. The highest shares in PC belonged to Sciuro-hypnum starkei $(30 \%)$, Polytrichum juniperinum (30\%) and the common forest species Pleurozium schreberi (8\%). Lichens were highly diverse with 17 species, mainly from the Cladonia genus. Thus, the end of the second experimental decade saw formation the forest community where sown plants of the first "intensive" restoration stage were normally replaced by forest species together with mosses and lichens.

On the 20th experiment year, soil pits on open area and in group of trees were excavated.

Soil pit №1 was dug in a group of trees (Betula pubescens, Larix sibirica). Ground cover (TPC 75\%) was found for the herbs (Avenella flexuosa, Solidago virgaurea, Alopecurus pratensis, Chamaenerion angustifolium, Orthilia secunda) and the mosses (Sciuro-hypnum starkei, Pleurozium schreberi). Moss cover was well developed (PC 60\%) with practically full-formed mossy litter.

A0 0-5 cm Loose layer of mossy litter, upper part contains weakly-decomposed and lower part stronger decomposed plant remnants with inclusions of sand, abundant roots.

A1 5-21 cm Sandy, grey-black, loose, inclusions of weakly- to well-decomposed plant remnants from outside peat (brought at the 1st restoration stage), many roots, transitional boundary is abrupt by color.

BC 21-35 cm Sand, grey-yellowish, with whitish spots, structureless, few roots, moist.

Soil pit №2 was made on open area. Herbaceous cover (TPC 100\%) was dominated by Alopecurus pratensis, Solidago virgaurea, Omalotheca sylvatica. There was a 2-cm-thick layer of dead grass on surface. Dead grass was the development base for the Brachythecium mosses and Sciuro-hypnum starkei.

A0 0-2(4) cm Loose layer of weakly-mean-decomposed plant remnants, dark-grey, sand inclusions in lower part, abundant roots.

A1 2(4)-21 cm Sandy, dark-grey to black, structureless, moist, abundant roots, inclusions of decomposed peat remnants brought at the 1st restoration stage, many rain worms, transitional boundary is abrupt by color.

BC 21-36 cm Sandy, grey-yellowish, with whitish spots, moist, upper part with single roots.

Agrochemical parameters of the studied soils (Table 7) provide evidence that the biogenicaccumulative layers (litter and humus horizons) have been formed on the 20th restoration year. Those horizons were marked through high content of organic carbon, nitrogen, exchangeable bases, and other biogenic elements. Humus of the biogenic-accumulative layer was dominated by humic substances (Table 10). 


\begin{tabular}{|c|c|c|c|c|c|c|c|c|c|c|c|c|}
\hline \multirow{2}{*}{$\begin{array}{l}\text { Horizon, } \\
\text { depth, cm }\end{array}$} & \multirow{2}{*}{$\begin{array}{l}\text { Corg. } \\
\text { total, } \%\end{array}$} & \multicolumn{4}{|c|}{ Humic acids } & \multicolumn{5}{|c|}{ Fulvic acids } & \multirow{2}{*}{$\begin{array}{l}\text { Non- } \\
\text { soluble } \\
\text { residue }\end{array}$} & \multirow{2}{*}{$\begin{array}{l}\mathrm{C}_{\mathrm{HA}} / \\
\mathrm{C}_{\mathrm{FA}}\end{array}$} \\
\hline & & 1 & 2 & 3 & $\sum$ & 1a & 1 & 2 & 3 & $\sum$ & & \\
\hline A1 3-13 & 4.2 & 20.94 & 11.76 & 20.7 & 53.4 & 3.18 & 14.7 & 6.24 & 9.88 & 34.0 & 12.6 & 1.57 \\
\hline A1 13-23 & 2.4 & 10.98 & 14.22 & 14.4 & 39.6 & 4.88 & 12.6 & 6.5 & 6.1 & 30.08 & 30.32 & 1.32 \\
\hline $23-28$ & 0.2 & 9.52 & 1.91 & 4.76 & 16.19 & 28.6 & 9.5 & 17.17 & 7.14 & 62.41 & 21.4 & 0.26 \\
\hline $28-45$ & 0.1 & 7.7 & 0.76 & 4.61 & 13.07 & 15.38 & 2.32 & 22.29 & 1.51 & 41.5 & 45.43 & 0.31 \\
\hline
\end{tabular}

Table 10. Fraction-group humus composition of organic-accumulative layer of the newlyformed soil on the 18th experiment year ( $\%$ of total content).

Consequently, the biological cycle of organic (plant) matter started at the "intensive" stage resulted in forest ecosystem formed to the 20th year as the integrity of two components, plant community (or biotic complex) and soil. Organic (plant) matter biological cycle restoration initiated active soil restoration visualized by formation of the biogenic-organicaccumulation layer. This layer's structure depends on plant community type. It determines the significance of soil as a system structure, capable of holding and accumulating plant nutrition elements and ensuring stable conditions for self-restoration of ecosystem. These properties are formed during the transformation processes of plant waste called humus formation, the main soil formation process (Ponomareva, Plotnikova, 1980).

Restoration of nature medium components is a complete process that functionally unifies biota with its habitat. Soil can be formed when technogenic substratum reaches some "critical" mass of plant material to start the biological cycle, including humus accumulation.

In the North, the process of self-restoring succession is a long-term process. To speed up (manage) the self-restoration process on post-technogenic bare areas, is to apply a complex of agrotechnical methods, i.e. fertilization, sowing perennial grasses, that is called an "intensive" restoration period. Accumulation of organic matter (plant remnants of perennial herbs etc.) in substratum, its transformation (humus formation) with help of zoo-microbe complex, accumulation and consequent assimilation of biogenic elements by plants provide favorable conditions for the next stage, forest ecosystem development.

The conducted study has evidenced the efficiency of agromethods ("intensive" stage) for speeding up the restoration process of forest ecosystem. It was demonstrated that the first experimental decade was already indicated by the most advanced restoration succession stage, i.e. formation the herbaceous community and its transition to forest community of quicklygrowing woody species under whose canopy conifers started growth. Transformation of herbaceous community and corresponding soil type during self-restoring succession in taiga zone into quickly-growing woody species stand is a normal process (Shennikov, 1964).

Acceleration in forest ecosystem formation becomes more prominent when comparing the study plot with the near self-restoring plot. On its 28th restoration year the TPC figure remained under $1 \%$ without woody plants and with active erosion signs. 


\section{Optimization the "nature restoration" methods}

As said above, the preliminary "intensive" stage ensures favorable substratum conditions for acceleration of woody layer self-restoration, replacement of herbaceous ecosystem by forest ecosystem. However, restoration of conifers proceeds slowly and under the canopy of quickly-growing deciduous (birch, asp) species. To further accelerate restoration of forest ecosystem on the second restoration stage, complex of methods was developed. These methods are to optimize restoration of conifers in woody layer of forest ecosystems in north taiga zone and consist in planting conifers simultaneously with agrotechnical treatments on the first ("intensive") restoration stage.

Another experiment on the territory of $8 \mathrm{~b}$ sand-pit was started, where Pinus sylvestris twoyear-old trees, traditionally used for restoration purposes, with open root system were planted with a planting density of 5000 individuals/ha. Herb mixture composition being sown on "intensive" stage included Poa pratensis, Festuca rubra, Festuca pratensis, Bromopsis inermis, Phleum pratense in proportion 1:1:1:1:1. Annual additional fertilization with complex mineral fertilizer was done during 4 years. By our data, only $30-40 \%$ of pine plantings remained alive on the second year and resisted few for the whole study period. The twoyear-old Pinus sylvestris plantings did not develop well on the "intensive" restoration stage with a height of 12-17 cm at the fifth year. Herb stand was already $90 \mathrm{~cm}$ high at that period of time with $80-90 \%$ TPC. So, the study has identified two-year-old Pinus sylvestris plantings with open root system not a promising material to be used on the "intensive" restoration stage of the "nature restoration" experiment. Herbaceous layer developed quicker than Pinus sylvestris plantings. Low growth rates did not allow the plantings to overgrow herb layer in a short period of time which was particularly responsible for their future underdevelopment.

Absolutely other results were obtained on usage the high-growth material, Pinus sylvestris wildlings about $50 \mathrm{~cm}$ high with a ground clot 30x30 cm. Planting density was 2500 individuals/ha. The same herb species as sown in the trial with two-year-old plantings were used. Additional fertilizing with complex mineral fertilizer (N45P45K45) was done every spring for 4 years.

By the observation results, the planted wildlings remained alive by almost $100 \% 5$ years afterwards (Table 11). High surviving rate of the plants was related to their sufficient height, planting with ground clot, and caring for 5 years.

\begin{tabular}{|l|l|l|l|l|}
\hline $\begin{array}{c}\text { Year(s) after } \\
\text { planting }\end{array}$ & $\begin{array}{c}\text { Survival rate, } \\
\%\end{array}$ & $\begin{array}{c}\text { Height, } \\
\mathrm{cm}\end{array}$ & \multicolumn{1}{|c|}{$\begin{array}{c}\text { Stem diameter, } \\
\mathrm{cm}\end{array}$} & $\begin{array}{c}\text { Crown diameter, } \\
\mathrm{cm}\end{array}$ \\
\hline 1 & 100 & $59.1 \pm 2.4$ & $1.1 \pm 0.1$ & $32.9 \pm 1.4$ \\
\hline 2 & 100 & $60.9 \pm 2.8$ & $1.5 \pm 0.2$ & $37.3 \pm 1.6$ \\
\hline 3 & 100 & $68.8 \pm 3.1$ & $1.7 \pm 0.1$ & $46.5 \pm 2.1$ \\
\hline 4 & 96 & $79.5 \pm 3.1$ & $1.8 \pm 0.1$ & $48.7 \pm 3.1$ \\
\hline 5 & 96 & $100.3 \pm 4.3$ & $2.1 \pm 0.2$ & $55.4 \pm 4.5$ \\
\hline
\end{tabular}

Table 11. Biometric parameters of Pinus sylvestris plants in optimization experiment (autumn observations). 
Beginning from the third planting year, Pinus sylvestris steadily increased in height and was over $20 \mathrm{~cm}$ high on the fifth year (Fig. 2).

On the fifth year the mean height of planted trees was about $1 \mathrm{~m}$, consequently, the trees showed high survival rate and well development.

Herbaceous cover on the experimental plot actively developed. On the third year TPC of herb layer was $30 \%$ and already $70-75 \%$ to the fourth-fifth year (Table 12). Herb layer practically lost such sown herbs as Festuca pratensis, soil moisture-dependent, and Trifolium pratense. The rhizome grasses Bromopsisinermis, Poa pratensis and the rhizome loose-bunch Festuca rubra remained. The latter species as least dependent of soil richness and moisture had the highest projective cover among sown herbs. New non-sown herbs appeared and were prevailed by Festuca ovina that normally grows in lichen pine forests. There were species typical of anthropogenically disturbed areas as Chamaenerion angustifolium, Equisetum arvense. The species Solidago virgaurea, Deschampsia cespitosa, Avenella flexuosa, usual for forest and meadow were fixed but were few in number. Forest sub-shrubs (Empetrum hermaphroditum, Vaccinium vitis-idaea) transported there within ground clot were identified. Moss cover started formation and included pioneer species (Table 12). The majority of newly-appeared species were single in number. The vivid species composition on the study plots characterized the plant community as young and unstable.

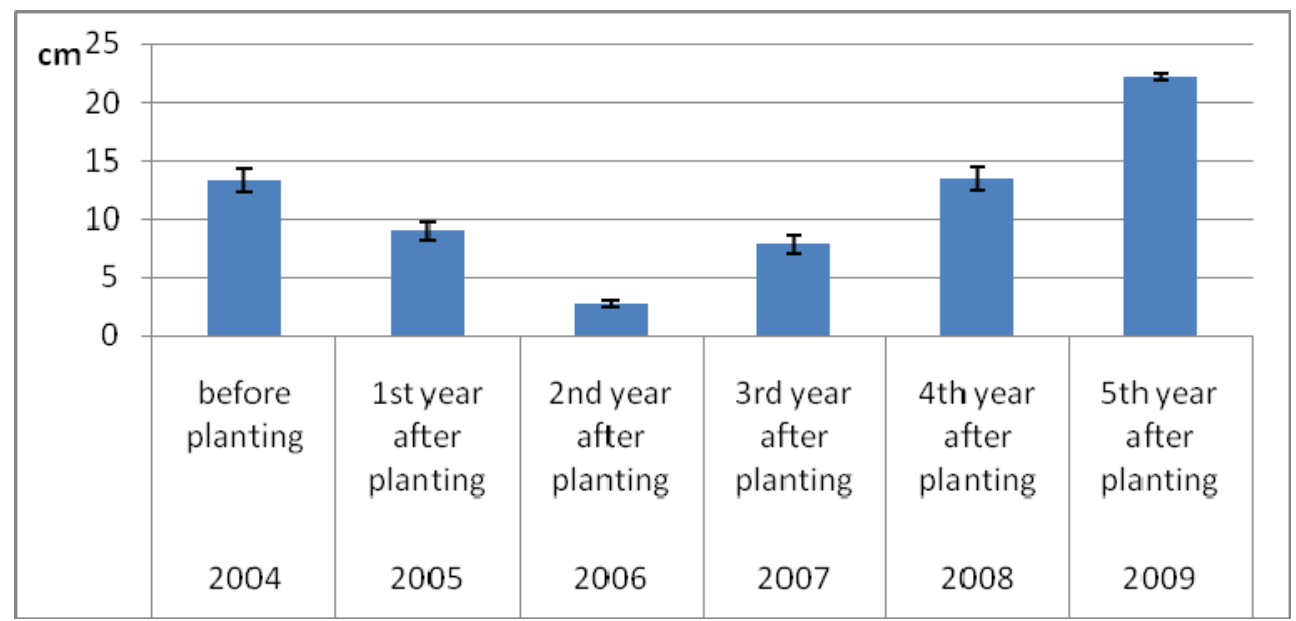

Fig. 2. Increment dynamics of Pinus sylvestris wildlings in optimization experiment. 


\begin{tabular}{|c|c|c|c|c|}
\hline \multirow[t]{2}{*}{ Species } & \multicolumn{4}{|c|}{$\begin{array}{c}\text { Number of years after experimental } \\
\text { start }\end{array}$} \\
\hline & 2 & 3 & 4 & 5 \\
\hline \multicolumn{5}{|l|}{ Sown herbs: } \\
\hline Bromopsis inermis (Leyss.) Holub & 2 & 6 & 10 & 7 \\
\hline Festuca pratensis Huds. & 1 & 3 & $<1$ & 2 \\
\hline Festuca rubra L. & 2 & 3 & 7 & 26 \\
\hline Phleum pratense L. & 4 & 7 & 5 & 5 \\
\hline Poa pratensis L. & 2 & 5 & 8 & 6 \\
\hline Trifolium pratense $\mathrm{L}$. & 1 & 1 & $<1$ & 1 \\
\hline \multicolumn{5}{|l|}{ Invasive species: } \\
\hline Avenella flexuosa (L.) Drey. & - & - & - & 1 \\
\hline Agrostis tenuis Sibth. & $<1$ & $<1$ & - & - \\
\hline Carex arctisibirica (Jurtz.) Czer. & - & $<1$ & $<1$ & $<1$ \\
\hline Chamaenerion angustifolium (L.) Scop & 3 & 2 & 4 & 1 \\
\hline Chenopodium album L. & - & $<1$ & - & - \\
\hline Crepis tectorum $\mathrm{L}$. & - & - & 1 & - \\
\hline Dactylis glomerata L. & - & - & $<1$ & 1 \\
\hline Deschampsia cespitosa (L.) Beauv. & - & - & $<1$ & $<1$ \\
\hline Empetrum hermaphroditum (Lange) Hagerup & $<1$ & $<1$ & $<1$ & $<1$ \\
\hline Equisetum arvense L. & 3 & 2 & 1 & $<1$ \\
\hline Festuca ovina L. & 10 & 16 & 41 & 20 \\
\hline Hieracium umbellatum L. & 1 & 3 & $<1$ & 2 \\
\hline Solidago virgaurea L. & $<1$ & $<1$ & $<1$ & $<1$ \\
\hline Tripleurospermum perforatum (Merat.) M.Lainz & $<1$ & $<1$ & - & - \\
\hline Vaccinium uliginosum L. & - & $<1$ & - & - \\
\hline Vaccinium vitis-idaea $\mathrm{L}$. & $<1$ & $<1$ & $<1$ & $<1$ \\
\hline \multicolumn{5}{|l|}{ Mosses } \\
\hline Ceratodon purpureus (Hedw.) Brid & $<1$ & $<1$ & $<1$ & 5 \\
\hline Polytrichum juniperinum Hedw. & $<1$ & $<1$ & 1 & $<1$ \\
\hline Polytrichum piliferum Hedw. & $<1$ & $<1$ & 1 & 1 \\
\hline Moss protonema & - & 21 & 32 & 30 \\
\hline Total projective cover & 30 & 48 & 75 & 70 \\
\hline Number of herb species & 18 & 18 & 17 & 17 \\
\hline Number of moss species & 3 & 3 & 3 & 3 \\
\hline
\end{tabular}

Table 12. Development characterization of herbaceous cover in the optimization experiment (projective cover by years, \%).

The changes in ground vegetation cover provoked changes in substratum. Substratum surface was identified for a loose layer of dead plant remnants (litter). On the fourth-fifth restoration year it became underlain by a weakly-compact soddy layer up to 3(5) cm thick. Slow dead plant material decomposition in the North causes slow organic carbon accumulation in substratum (Table 13, Fig. 3). This fact was proven by other scientists (Abakumov, 2008). There is an existed positive trend in content of biogenic elements (Figs. $4,5,6)$ related to the already started organic matter biological cycle. 


\begin{tabular}{|c|c|c|c|c|c|c|c|c|}
\hline \multirow{2}{*}{$\begin{array}{l}\text { Plot, } \\
\text { № }\end{array}$} & \multirow{2}{*}{$\begin{array}{l}\text { Sampling } \\
\text { depth, cm }\end{array}$} & \multirow{2}{*}{$\mathrm{pH}_{\text {water }}$} & \multirow{2}{*}{$C, \%$} & $\mathrm{~N}_{\text {hydr. }}$ & $\mathrm{P}_{2} \mathrm{O}_{5}$ & $\mathrm{~K}_{2} \mathrm{O}$ & $\mathrm{Ca}^{2+}$ & $\mathrm{Mg}^{2+}$ \\
\hline & & & & \multicolumn{3}{|c|}{$\mathrm{Mg} / 100 \mathrm{~g}$ a.d.s. } & \multicolumn{2}{|c|}{$\mathrm{mM} / 100 \mathrm{~g}$ a.d.s. } \\
\hline \multicolumn{9}{|c|}{ initial substratum } \\
\hline \multirow{2}{*}{ control } & $0-10$ & 5.7 & 0.1 & 0.2 & 6.6 & 2.1 & 0.6 & 0 \\
\hline & $20-30$ & 5.7 & 0.1 & 0.4 & 8.1 & 2.2 & 0.5 & 0.1 \\
\hline trial & $0-10$ & 6.5 & 0.2 & 0.3 & 5.7 & 2.9 & 0.9 & 0.0 \\
\hline \multicolumn{9}{|c|}{ on the second year after planting } \\
\hline \multirow{2}{*}{ control } & $0-5$ & 5.8 & 0.2 & 0.3 & 6.3 & 3.8 & 1.1 & 0.5 \\
\hline & $5-10$ & 5.7 & 0.2 & 0.3 & 6.5 & 3.2 & 0.9 & 0.3 \\
\hline \multirow{2}{*}{ trial } & $0-5$ & 6.1 & 0.3 & 1.3 & 5.6 & 3.0 & 1.1 & 0.1 \\
\hline & $5-10$ & 6.1 & 0.2 & 0.3 & 3.4 & 1.8 & 1.0 & 0.4 \\
\hline \multicolumn{9}{|c|}{ on the fourth year after planting } \\
\hline \multirow{2}{*}{ control } & $0-5$ & 5.8 & 0.1 & 0.4 & 10.5 & 4.7 & 0.6 & 0.1 \\
\hline & $5-15$ & 5.9 & 0.1 & 0.3 & 9.5 & 2.7 & 0.8 & 0.1 \\
\hline \multirow{3}{*}{ trial } & $0-3$ & 6.1 & 0.3 & 1.0 & 16.2 & 7.4 & 0.8 & 0.1 \\
\hline & 3-15 & 6.0 & 0.1 & 0.2 & 5.7 & 3.9 & 1.3 & 0.2 \\
\hline & $15-30$ & 6.4 & 0.1 & 0.5 & 5.6 & 2.5 & 1.3 & 0.4 \\
\hline \multicolumn{9}{|c|}{ on the fifth year after planting } \\
\hline \multirow{4}{*}{ control } & $0-5$ & 5.3 & 0.1 & 0.4 & 7.2 & 5.1 & 0.4 & 0.3 \\
\hline & $5-10$ & 5.2 & 0.1 & 0.3 & 7.9 & 5.4 & 0.3 & 0.2 \\
\hline & $10-20$ & 5.2 & 0.2 & 0.4 & 6.9 & 5.5 & 0.3 & 0.2 \\
\hline & $20-30$ & 5.2 & 0.1 & 0.3 & 7.0 & 5.3 & 0.3 & 0.2 \\
\hline \multirow{4}{*}{ trial } & $0-5$ & 5.1 & 0.2 & 1.5 & 11.1 & 13.4 & 0.7 & 0.2 \\
\hline & $5-10$ & 5.5 & 0.1 & 0.7 & 5.0 & 4.7 & 0.9 & 0.3 \\
\hline & $10-15$ & 6.0 & 0.1 & 1.0 & 6.5 & 4.1 & 0.9 & 0.3 \\
\hline & $15-30$ & 6.0 & 0.1 & 0.7 & 5.0 & 3.4 & 0.7 & 0.3 \\
\hline \multicolumn{9}{|c|}{ on the sixth year after planting } \\
\hline \multirow{4}{*}{ control } & $0-5$ & 5.2 & 0.1 & 0.4 & 7.2 & 2.2 & 0.6 & 0.2 \\
\hline & $5-10$ & 5.2 & 0.1 & 0.2 & 7.9 & 2.9 & 0.8 & 0.2 \\
\hline & $10-20$ & 5.2 & 0.1 & 0.2 & 8.8 & 3.2 & 0.8 & 0.3 \\
\hline & $20-30$ & 5.3 & 0.1 & 0.3 & 9 & 2.9 & 0.8 & 0.4 \\
\hline \multirow{5}{*}{ trial } & Ад 0-2 & 5.2 & 0.2 & 2.2 & 32.1 & 10.2 & 0.6 & 0.3 \\
\hline & АдА $\mathrm{A}_{1} 2-5$ & 5.4 & 0.1 & 2.5 & 13.8 & 12.3 & 0.4 & 0.1 \\
\hline & $\mathrm{A} / / 5-10$ & 5.4 & 0.2 & 0.7 & 9.5 & 10.1 & 0.6 & 0.2 \\
\hline & AB 10-20 & 5.6 & 0.2 & 1.9 & 12.1 & & 0.8 & 0.2 \\
\hline & B $20-30$ & 6.1 & 0.1 & 0.3 & 9.1 & 2.9 & 1.3 & 0.3 \\
\hline
\end{tabular}

Table 13. Changes in substratum agrochemical parameters in the optimization experiment. 


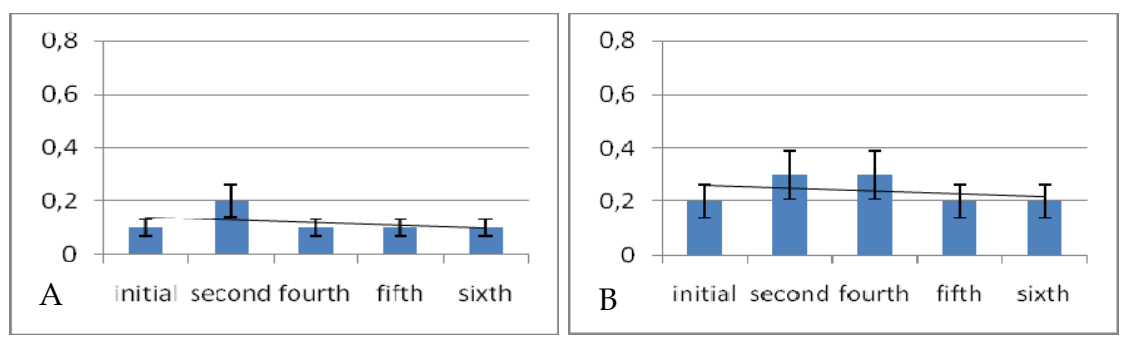

Fig. 3. Organic carbon content dynamics (\%) by years in upper substrata layer in the background (A) and experimental (B) plots.
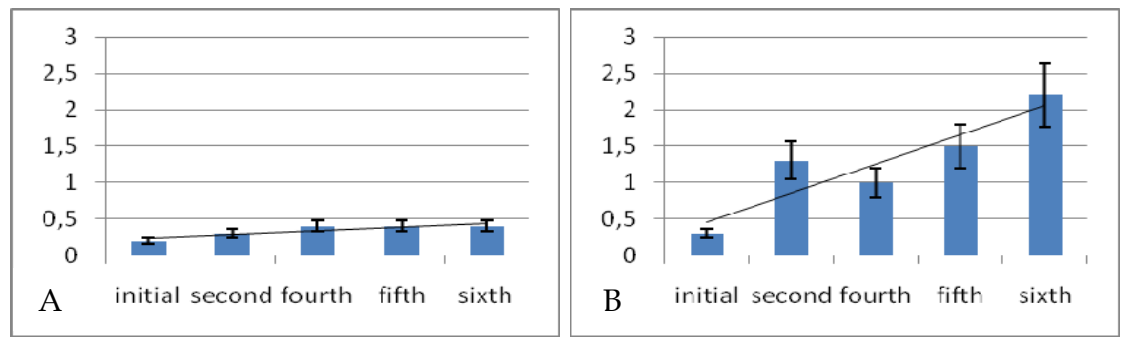

Fig. 4. Hydrolizable nitrogen content dynamics (mg/100 g a.d.s.) in upper substrata layer in the background (A) and experimental (B) plots.
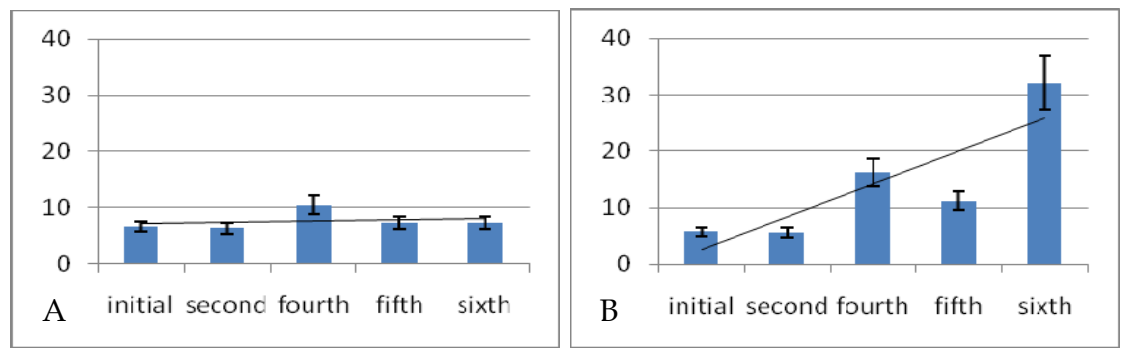

Fig. 5. Phosphorus oxide content dynamics (mg/100 g a.d.s.) in upper substrata layer in the background (A) and experimental (B) plots.
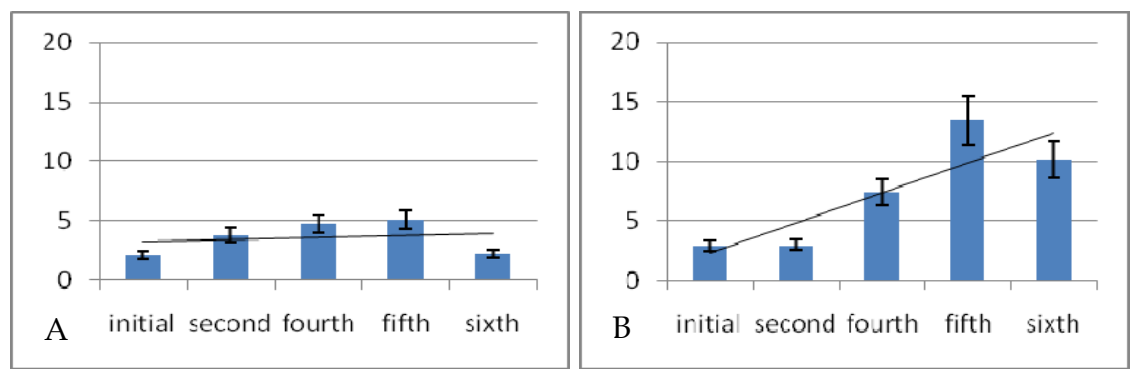

Fig. 6. Potassium oxide content dynamics (mg/100 g a.d.s.) in upper substrata layer in the background (A) and experimental (B) plots. 
By the obtained experiment results, usage of intensive agromethods combined with highquality planting material, big-size Pinus sylvestris plantings with ground clot, ensures the high survival rate and active growth of the planted conifers together with herbaceous layer formation. This is important because it causes simultaneous transformation of technogenic substratum and formation of soil as a forest ecosystem component. Soil development is behind plant community development; soil formation in morphological and chemical senses can be only after accumulation of some "critical" plant mass and its transformation products (humus) in substratum.

The popular opinion about the necessity of herb cover destruction while planting of trees in order to improve their growth (competition for nutrients) appeared to be questionable, especially on usage of big-size plantings.

This experiment has shown possibility of accelerated formation of forest ecosystem already on the first ("intensive") restoration stage. Further observations will allow for more recommendations on the optimized experiment.

\section{Conclusion}

The usage of two-stage "nature restoration" approach ensures active self-restoration of forest ecosystem in far-north taiga (on forest distribution border). It is vital to mention that "nature restoration" conception and its application in practice widen the traditional sense of the term "recultivation" not only by geographical point but also by understanding the functional interdependence between ecosystem components linked together by organic matter biological cycle.

Taking into consideration the serious ecological situation not only in the North, very significant is to revise our opinion on recultivation. Common sense of recultivation is returning lands into repeated agricultural usage. But nowadays there is the need to revise this term. In this view, "nature restoration" conception has a deep sense with its system approach aiming at accelerating restoration of nature ecosystems on disturbed areas exerting important biosphere functions. The system of "nature restoration" can be widely used, including tropical forests, however with some corrections in respect to particular climatic conditions. It is important to mention that oil-polluted lands' restoration at "intensive" stage requires usage of special purifying preparations followed by agrothechnical methods. In view of progressive development of economics, intensive "nature exploitation" should be accompanied by fullscale accelerated (managed) restoration of zonal ecosystems on disturbed areas, proportional to disturbance extent. Imbalance in "human-nature" system produces ecological critical situations (Ecological principles..., 2010).

In relation to the above-said, in newly-published work of K.S. Losev (2010) it says that only some part of natural ecosystems on Earth can be replaced by artificial ecosystems (agrarian or technogenic) without hampering the biological regulation mechanism, responsible for biosphere balance. He calls territories under such artificial ecosystems as ecological (economic) biosphere parts. The importance of natural ecosystems as a biosphere stability factor is now underestimated. The aftereffects cause a row of nature medium changes visualized in climate change, progressive environmental pollution, soil poorness, poor human's health, etc. There is a need of changing the understanding and treat the nature with more responsibility for conservation the environment and wild world in its initial 
diversity. One simple rule should be followed - whatever we took from nature (disturbed) we are to recover by means of additional work and financial expenses. This is the closest link between ecology and economics.

This work was financially supported by RAS Presidium Program № 23 "Biological diversity" within the topic "Biodiversity formation rules of plant communities in restoring and transforming ecosystems in different types of technogenic objects in the North-East of European Russia", № 09-П-4-1028.

\section{References}

[1] Abakumov E.V. Accumulation and transformation of organic matter on different-aged waste piles of sand pit / / Soil Science J., 2008. №8. p.955-963

[2] Agrochemical methods of soil study. M.: USSR Science Academy Publishing House, 1960. p.556

[3] Archegova I.B. Nature restoration efficient system - the base of promising nature management in the Far North, Syktyvkar. 1998. 12 p. (Scientific reports / Komi SC UrD RAS; Iss. 412).

[4] Ecological principles of nature management and nature restoration in the North. Coauthorship. Editor-in-chief I.B. Archegova. Syktyvkar. 2009. p.176

[5] Field geobotany. M.-L.: Nauka, 1964. p.532

[6] Guidance on humus content and composition estimation in (mineral and peat) soils. Leningrad, 1975. p.106

[7] Larin V.B., Pautov Yu.A., Pruchkin V.D. Stable development strategy of forest region in the North // Finno-Ugric World: Nature Health Status and Regional Strategy of Environment Protection: Proc. Int. Conf. Syktyvkar, 2000. p.93-98

[8] Losev K.S. Myths and mistakes in ecology. M.: Nauchnyi mir, 2010. p.224

[9] Losev K.S., Mnatsakanyan R.A., Dronin N.M. Consumption of renewed resources: ecological and social-economic consequences (global and regional aspects). M.: GEOS, 2005, p.158

[10] Ogievskiy V.V., Khirov A.A. Observation and investigation of forest cultures. M.: Lesnaya promyshlennost', 1964. p.48

[11] Parfenyuk V.I. The method of oil-polluted soil recultivation. Patent №20009626 (patented in the State Register of Inventions February 19, 1991).

[12] Podzolic soils of the central and eastern parts of the European USSR. - L., 1981. p. - 200

[13] Ponomareva V.V. Forest as elluvial-resistant vegetation type / / Botanical Journal, Band 55, № 11. 1970. p.1585-1595.

[14] Ponomareva V.V., Plotnikova T.A. Humus and soil formation (study methods and results). L.: Nauka. 1980. p.222

[15] Scientific-applied reference book on the USSR climate. Series 3, Perennial data. P. 1-6. Iss. 1. Archangelskaya and Vologodskaya regions, Komi ASSR. L.: Hidrometeoizdat, 1989, book 1. p.483

[16] Semenkova I.G., Sokolova E.C. Forest phytopathology. M.: Ecology, 1992. p.352

[17] Shennikov A.P. Introduction to geobotany. - L.: Leningrad Uni. Publishing House, p.447

[18] Theory and practice of soil chemical analysis / Ed. by Vorobyeva L.A. M.: GEOS, 2006. p.400

[19] Yudin Yu.P. Geobotanical zoning // Productive forces of the Komi ASSR, M.-L. 1954, Band. 3, P. 1. p.323-359 


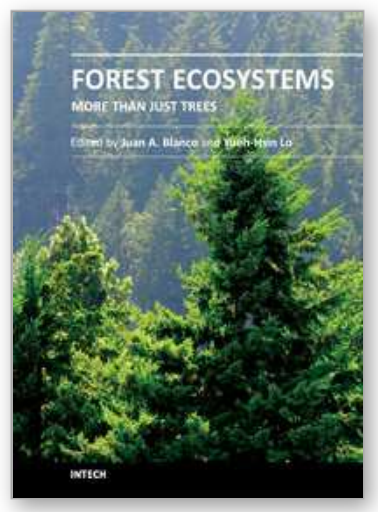

\section{Forest Ecosystems - More than Just Trees}

Edited by Dr Juan A. Blanco

ISBN 978-953-51-0202-1

Hard cover, 464 pages

Publisher InTech

Published online 07, March, 2012

Published in print edition March, 2012

The common idea for many people is that forests are just a collection of trees. However, they are much more than that. They are a complex, functional system of interacting and often interdependent biological, physical, and chemical components, the biological part of which has evolved to perpetuate itself. This complexity produces combinations of climate, soils, trees and plant species unique to each site, resulting in hundreds of different forest types around the world. Logically, trees are an important component for the research in forest ecosystems, but the wide variety of other life forms and abiotic components in most forests means that other elements, such as wildlife or soil nutrients, should also be the focal point in ecological studies and management plans to be carried out in forest ecosystems. In this book, the readers can find the latest research related to forest ecosystems but with a different twist. The research described here is not just on trees and is focused on the other components, structures and functions that are usually overshadowed by the focus on trees, but are equally important to maintain the diversity, function and services provided by forests. The first section of this book explores the structure and biodiversity of forest ecosystems, whereas the second section reviews the research done on ecosystem structure and functioning. The third and last section explores the issues related to forest management as an ecosystem-level activity, all of them from the perspective of the other parts of a forest.

\section{How to reference}

In order to correctly reference this scholarly work, feel free to copy and paste the following:

Irina Likhanova and Inna Archegova (2012). Restoration of Forest Ecosystems on Disturbed Lands on the Northern Forest Distribution Border (North-East of European Russia), Forest Ecosystems - More than Just Trees, Dr Juan A. Blanco (Ed.), ISBN: 978-953-51-0202-1, InTech, Available from:

http://www.intechopen.com/books/forest-ecosystems-more-than-just-trees/restoration-of-forest-ecosystemson-disturbed-lands-on-the-northern-forest-distribution-border-north

\section{INTECH}

open science | open minds

\section{InTech Europe}

University Campus STeP Ri

Slavka Krautzeka 83/A

51000 Rijeka, Croatia

Phone: +385 (51) 770447

\section{InTech China}

Unit 405, Office Block, Hotel Equatorial Shanghai

No.65, Yan An Road (West), Shanghai, 200040, China

中国上海市延安西路65号上海国际贵都大饭店办公楼 405 单元

Phone: +86-21-62489820 
Fax: +385 (51) 686166

Fax: +86-21-62489821

www.intechopen.com 
(C) 2012 The Author(s). Licensee IntechOpen. This is an open access article distributed under the terms of the Creative Commons Attribution 3.0 License, which permits unrestricted use, distribution, and reproduction in any medium, provided the original work is properly cited. 